

Figure 1. The first experimental $(\mathrm{Okl})$ reciprocal space plane of $\mathrm{BaCuSi}_{2} \mathrm{O}_{6 \pm \delta}(1 \mathrm{bar})$ at $4.3 \mathrm{~K}$.

Keywords: growth with oxygen partial pressure, phase transition, spin dimer compounds

\section{MS26-P7 Complete decoupling of magnetic order and superconducitvity in a conventional superconductor}

\author{
Daniel Mazzone
}

email: daniel.mazzone@psi.ch

Conflicting electron states at the Fermi surface usually prevent a harmonic coexistence of superconductivity and magnetism in conventional superconductors. In order to improve the understanding of the interaction between superconductivity and magnetism a model material with missing competition between the two phenomena is required. In $\mathrm{Pr}_{2} \mathrm{Pt}_{3} \mathrm{Ge}_{5}$ we discovered a system, in which multiple magnetic orders and superconductivity not only coexist, but do not compete [1]. The systems displays a relatively high transition temperature $T=7.8 \mathrm{~K}$ and two antigerromagnetic transitions $T_{N I}=3.4$ and $T_{N 2}=4.1 \mathrm{~K}$ with a commensurate and an incommensurate canted structure $[1,2]$. We studied the superconducting and magnetic properties of he compound by means of neutron diffraction, magnetization and transport measurements. The interpenetrating $H T$-phase diagrams, representing the refined magnetic structures, preclude any direct competition between the superconducting and magnetic phases. We propose that different sheets oft he Fermi surface are involved in the magnetic and superconducting exchange interactions.

\section{Reference:}

[1] D. G. Mazzone et al., arxiv:1508.02492015 (2015).

[2] N. H. Sung et al., PRB 86, 224507 (2012).

Keywords: magnetic structure, superconductivity 